\title{
TALE-light imaging reveals maternally guided, H3K9me2/3-independent emergence of functional heterochromatin in Drosophila embryos
}

\author{
Kai Yuan and Patrick H. O'Farrell \\ Department of Biochemistry, University of California at San Francisco, San Francisco, California 94158, USA
}

\begin{abstract}
Metazoans start embryogenesis with a relatively naïve genome. The transcriptionally inert, late-replicating heterochromatic regions, including the constitutive heterochromatin on repetitive sequences near centromeres and telomeres, need to be re-established during development. To explore the events initiating heterochromatin formation and examine their temporal control, sequence specificity, and immediate regulatory consequence, we established a live imaging approach that enabled visualization of steps in heterochromatin emergence on specific satellite sequences during the mid-blastula transition (MBT) in Drosophila. Unexpectedly, only a subset of satellite sequences, including the 359-base-pair (bp) repeat sequence, recruited HP1a at the MBT. The recruitment of HP1a to the 359-bp repeat was dependent on HP1a's chromoshadow domain but not its chromodomain and was guided by maternally provided signals. HP1a recruitment to the 359 -bp repeat was required for its programmed shift to later replication, and ectopic recruitment of HP1a was sufficient to delay replication timing of a different repeat. Our results reveal that emergence of constitutive heterochromatin follows a stereotyped developmental program in which different repetitive sequences use distinct interactions and independent pathways to arrive at a heterochromatic state. This differential emergence of heterochromatin on various repetitive sequences changes their replication order and remodels the DNA replication schedule during embryonic development.
\end{abstract}

[Keywords: late replication; heterochromatin formation; satellite sequence; mid-blastula transition; HP1a; Drosophila] Supplemental material is available for this article.

Received September 18, 2015; revised version accepted January 26, 2016.

Very early in metazoan embryogenesis, epigenetic reprogramming remodels nucleosomes, changes histone modification patterns, and, in many organisms, refashions DNA methylation. Active mechanisms, which are most heavily investigated in mammals, along with the passive dilution effect caused by the rapid cell divisions characteristic of many early embryos, are thought to reset the newly formed zygotic genome to a relatively naïve state to restore totipotency (Fadloun et al. 2013; Farrell and O'Farrell 2014). As the embryo continues developing, the epigenetic constraints are progressively restored and thus canalize the cells toward distinct cell fates. Although it lies at the heart of developmental programing, we have only rudimentary ideas about how embryonic restoration of epigenetic marks is controlled.

Selective silencing of the genome by heterochromatin formation is one of the most important epigenetic events (Beisel and Paro 2011). In multicellular organisms, two types of heterochromatin exist. Constitutive heterochro-

Corresponding authors: ofarrell@cgl.ucsf.edu, kai.yuan@ucsf.edu Article published online ahead of print. Article and publication date are online at http://www.genesdev.org/cgi/doi/10.1101/gad.272237.115. matin, characterized by enrichment of dimethylated or trimethylated $\mathrm{H} 3 \mathrm{~K} 9$ (H3K9me2/3) and $\mathrm{HP} 1 \mathrm{a}$, silences genomic regions enriched with tandem repeats of DNA motifs (also known as satellite sequences) and remnants of transposable elements (TEs) (Elgin and Reuter 2013), whereas facultative heterochromatin represses selective domains of euchromatin in particular cells, thereby participating in cell fate determination during development. In early embryos, most signatures of both types of heterochromatin are erased and have to be re-established de novo. How to deploy these powerful silencing systems accurately is a developmental challenge. Here, by focusing on the formation of the constitutive heterochromatin on different satellite sequences in Drosophila embryos, we start to unveil the molecular process of transmission and re-establishment of epigenetic regulation during embryogenesis.

(C) 2016 Yuan and O'Farrell This article is distributed exclusively by Cold Spring Harbor Laboratory Press for the first six months after the full-issue publication date (see http://genesdev.cshlp.org/site/misc/terms.xhtml). After six months, it is available under a Creative Commons License (Attribution-NonCommercial 4.0 International), as described at http:// creativecommons.org/licenses/by-nc/4.0/. 
Like many other organisms, Drosophila begins embryonic development with rapid cell divisions (Farrell and O'Farrell 2014). Following the first gonomeric division, during which the male and female pronuclei meet, the zygotic nuclei undergo seven mitotic divisions (cycles 2-8) that are perhaps the shortest cell cycles ever documented (8.6 min for every division), resulting in a shell of nuclei inside a shared cytoplasm-the syncytium. At cycle 9, this shell of nuclei migrates to the surface of the embryo and forms the blastoderm. The nuclei then continue dividing rapidly another four times (cycles 10-13), with progressively increased interphase durations from $\sim 9 \mathrm{~min}$ in cycle 10 to $14 \mathrm{~min}$ in cycle 13 . This commitment to the cell cycle quickly expands the number of zygotic nuclei. Many other cellular processes and developmental events are deferred until the slowing of the cell cycle at cycle 14. The interphase of cycle 14 is abruptly lengthened to $\geq 70$ min depending on the spatial position of the cell in the embryo. In this lengthened interphase 14, cortical nuclei are cellularized, many maternal mRNAs and proteins are degraded, and zygotic transcription is fully activated. The embryonic development thus switches from maternal to zygotic programming (Lee et al. 2014). This major embryonic transformation is called the mid-blastula transition (MBT).

Various satellite sequences in somatic cells are packaged into constitutive heterochromatin, which has unique attributes, including high compaction, enrichment of $\mathrm{H} 3 \mathrm{~K} 9 \mathrm{me} 2 / 3$ and $\mathrm{HP} 1 \mathrm{a}$, transcriptional quiescence, and late replication. Most of these attributes are absent in the preblastoderm embryos, and the satellite sequences seem to take on these features successively as the embryo develops. Analysis of the C-banding patterns of the mitotic chromosomes (Vlassova et al. 1991) as well as assessment of the volume of a particular satellite sequence (Shermoen et al. 2010) suggest that satellite DNA becomes compacted prior to the blastoderm stage. However, neither the establishment of H3K9 methylation in heterochromatic regions nor the H3K4 methylation in euchromatic regions was observed until interphase 14 (Rudolph et al. 2007). Using a heat-shock-driven lacZ transgene inserted near satellite sequences, it was reported that the silencing activity of constitutive heterochromatin was first detectable in the gastrulating embryo, which is approximately in G2 of interphase 14 (Lu et al. 1998). In addition, BrdU or dUTP incorporation experiments revealed that satellite sequences became late-replicating in interphase 14 (Edgar and O'Farrell 1990; Shermoen et al. 2010). These observations imply that constitutive heterochromatin is matured around the time of MBT. The early embryonic formation of constitutive heterochromatin seems to play a unique role in long-term programming of gene expression, as selectively compromising HP1 function in early embryos by genetic manipulations influenced transcriptional silencing activity of heterochromatin in adults (Gu and Elgin 2013).

One caveat of the studies described above is the lack of spatial resolution. Constitutive heterochromatin contains many compositionally different satellite sequences located at distinct genomic positions. Without analyzing the behavior of each individual satellite sequence, our understanding of constitutive heterochromatin formation would be oversimplified. Here, by using the recently developed transcription activator-like effector (TALE)-light method (Yuan et al. 2014), we characterized the formation of heterochromatin on satellite sequences in Drosophila early embryos at unprecedented spatial-temporal resolution. We observed, at the time of MBT, a stereotyped program of introduction of molecular markers of heterochromatin on some repetitive sequences (359 base pairs [bp]) but not on the others (1.686). The resultant change of local chromatin landscape altered the order of replication of those repeats. Moreover, we interrogated the molecular mechanism of heterochromatinization of the 359-bp repeat and uncovered that signals from the mother guided the initial accumulation of HPla at the 359-bp loci independently of the histone mark H3K9me2/3. This study highlights the sophistication of the programs that introduce heterochromatic features to different regions of the genome within a nucleus and generates new insight into the inheritance of constitutive heterochromatin.

\section{Results}

Establishment of repressive histone modifications at the $M B T$

Previously, we observed that the replication of one satellite sequence, the 359-bp repeat, was only moderately delayed at the onset of late replication in interphase 14 but shifted to a much later time in interphase 15 (Fig. 1A; Shermoen et al. 2010; Yuan et al. 2014). We wanted to probe the mechanism of this selective sudden delay in replication and reasoned that local epigenetic changes might be involved. DNA methylation is absent in Drosophila melanogaster early embryos (Zemach et al. 2010), and histone modifications play a key role in early embryonic epigenetic reprogramming. We thus analyzed several representative histone modifications during the MBT.

While there was almost no detectable immunostaining with antibodies targeting $\mathrm{H} 3 \mathrm{~K} 9 \mathrm{me} 2$ or $\mathrm{H} 3 \mathrm{~K} 9 \mathrm{me} 3$ prior to interphase 13 , staining for $\mathrm{H} 3 \mathrm{~K} 9 \mathrm{me} 2$ and $\mathrm{H} 3 \mathrm{~K} 9 \mathrm{me} 3$ increased dramatically in interphase 14, with increases in H3K9me2 slightly preceding H3K9me3. This staining localized to the apical pole of nuclei where the pericentric sequences surround the clustered centromeres (Fig. 1B). Acetylation of $\mathrm{H} 3 \mathrm{~K} 9$ (H3K9ac), which is exclusive of H3K $9 \mathrm{me} 2 / 3$ and marks euchromatin on chromosome arms, was also undetected in the early embryo but appeared in cycle 12 (Supplemental Fig. S1A). Interestingly, staining for $\mathrm{H} 3 \mathrm{~K} 27 \mathrm{ac}$ as well as $\mathrm{H} 4 \mathrm{ac}$ was readily detectable as early as in the preblastoderm embryos (Supplemental Fig. S1C,D), but its level decreased slightly in mid-late interphase 14 when H3K27me3 started to appear (Supplemental Fig. S1B).

In summary (Fig. 1C), during the early embryonic development, histone acetylation appears early, accompanying the rampant DNA replication and the gradual activation of the zygotic genome (Li et al. 2014). On the other hand, the repressive histone modifications $\mathrm{H} 3 \mathrm{~K} 9 \mathrm{me} 2 / 3$ 

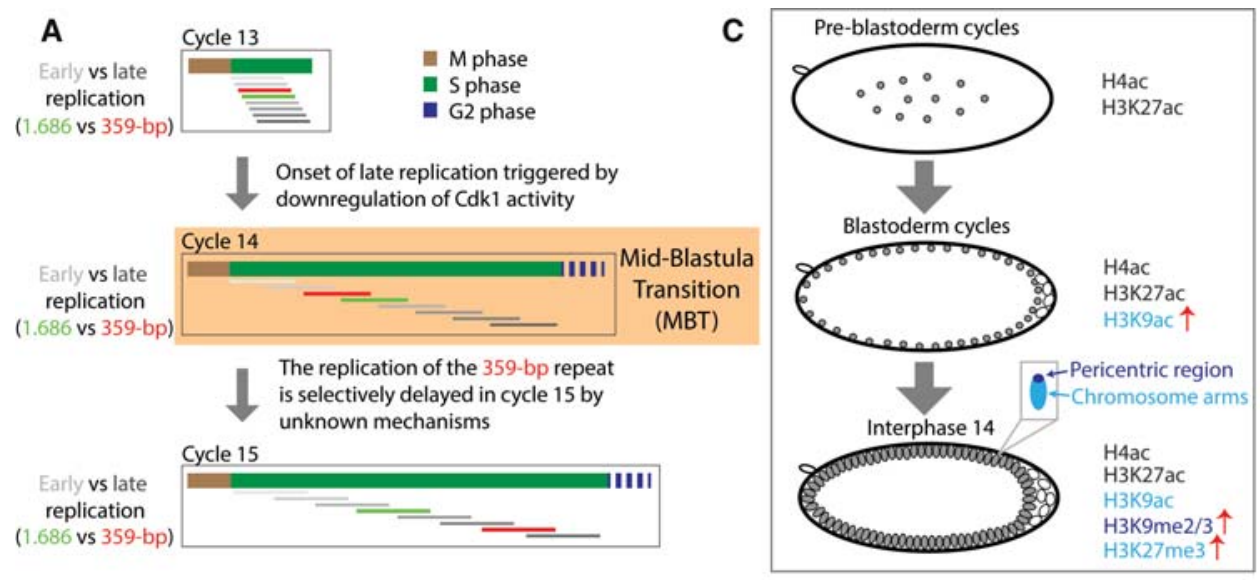

B

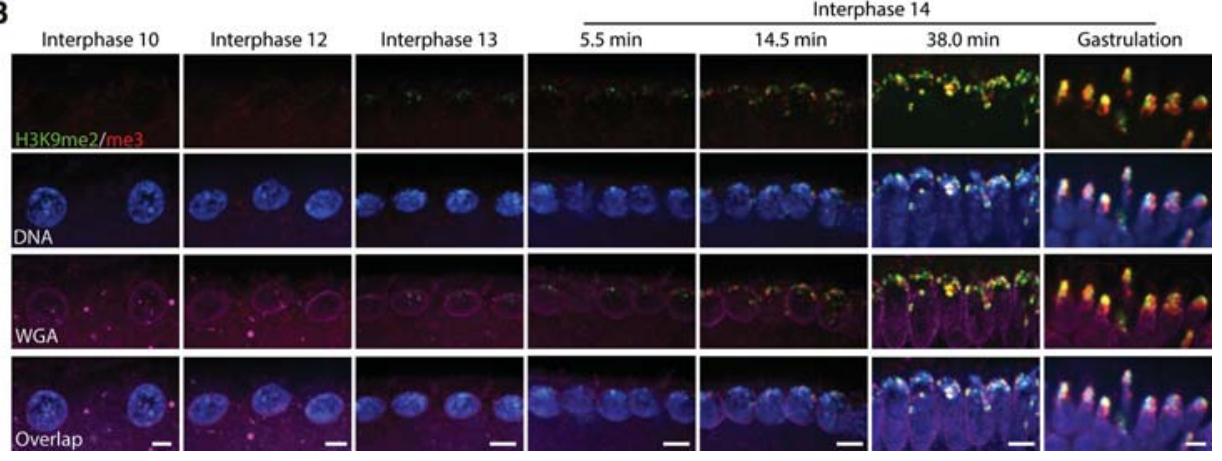

Figure 1. Onset of repressive histone modifications in interphase 14. (A) Approximations show replication timing of early-replicating (light-gray bars) and late-replicating (dark-gray bars) sequences in the interphases around the MBT. Green and red bars represent the replication timing of two particular repetitive sequences, 1.686 and $359 \mathrm{bp}$, respectively. Note that after the global onset of late replication in cycle 14, these two repeats switch order in replication in cycle 15 due to the much delayed replication of the 359-bp repeat. (B) The emergence of $\mathrm{H} 3 \mathrm{~K} 9 \mathrm{me} 2$ or $\mathrm{H} 3 \mathrm{~K} 9 \mathrm{me} 3$ in D. melanogaster early embryos. The antibody stainings of $\mathrm{H} 3 \mathrm{~K} 9 \mathrm{me} 2$ and $\mathrm{H} 3 \mathrm{~K} 9 \mathrm{me} 3$ are shown in green and red, respectively. All of the images were captured and processed using the same microscopic settings so that the fluorescent intensities faithfully reflect the relative quantities of the indicated modifications. Wheat germ agglutinin (WGA)-stained nuclear membrane is shown in magenta, and Hoechst-labeled DNA is in blue. The bottom rows show the staining for methylation in combination with DNA, wheat germ agglutinin, and both. The estimated developmental time, determined by either internuclear distance for embryos in the syncytial blastoderm stage or the length of the nuclei for embryos in cycle 14, is marked at the top. Bars, $5 \mu \mathrm{m}$. (C) A graphic summary showing the emergence of different histone modifications during $D$. melanogaster early embryonic development. In addition to the difference in their temporal appearance, different histone modifications show distinct spatial distribution (magnified nucleus). H3K9me2/3 is concentrated at the pericentric regions (dark blue), whereas $\mathrm{H} 3 \mathrm{~K} 9 \mathrm{ac}$ and $\mathrm{H} 3 \mathrm{~K} 27 \mathrm{me} 3$ are along the chromosome arms (light blue). The pole cells (germline precursors, at the posterior/right pole) show a distinct behavior.

and H3K27me3 do not significantly accumulate until the much prolonged interphase 14 , suggesting that the slowing of cell cycle at the MBT provides sufficient time for the establishment of repressive histone modifications.

\section{Different repetitive sequences acquire distinct chromatin features at the MBT}

Various highly repetitive sequences that occupy $>20 \%$ of the $D$. melanogaster genome are almost always heterochromatic and comprise "constitutive heterochromatin" (Wei et al. 2014). The appearance of H3K9me $2 / 3$ in interphase 14 marks a step in embryonic formation of constitutive heterochromatin. To probe whether each type of repeat sequence responds similarly to the developmental cues, we analyzed the emergence of histone modifications at the subheterochromatic level on different satellite sequences.

We previously developed protein probes called TALElight that can be programmed to recognize a given DNA sequence (Fig. 2A). Injection of a TALE-light at an optimal concentration highlighted particular satellite sequences live without disrupting embryogenesis (Supplemental Movie S1). Similarly, staining of fixed embryos with the TALE-light lit up the target sequences (Supplemental Fig. S2A). The dynamics of the mitotic chromosome seemed to affect the TALE-light's recognition of its target, as the TALE-light signal went down during mitosis in live embryos but not in fixed samples (Supplemental Fig. S2B).

We made TALE-lights that recognize two major satellite sequences in the $D$. melanogaster genome: 359-bp and 1.686 (Yuan et al. 2014). Costaining of the TALE-light and anti-histone 3 antibody suggested that these 
A

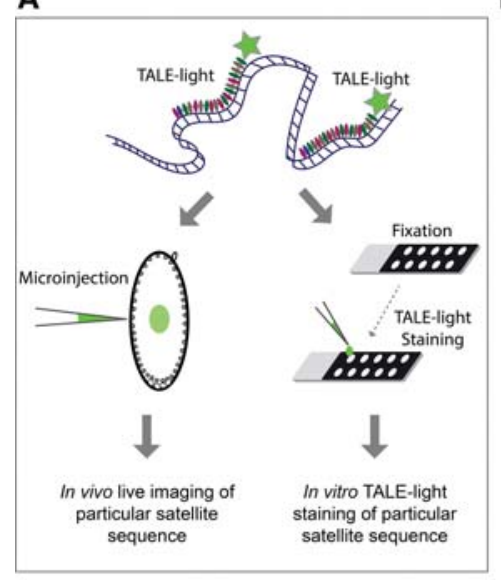

C 30.0 min into interphase 14

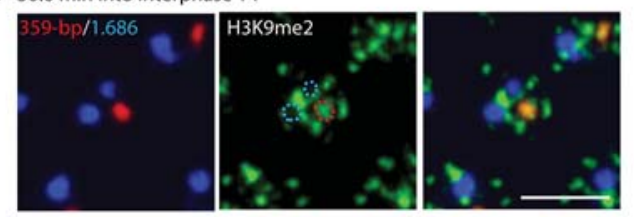

E

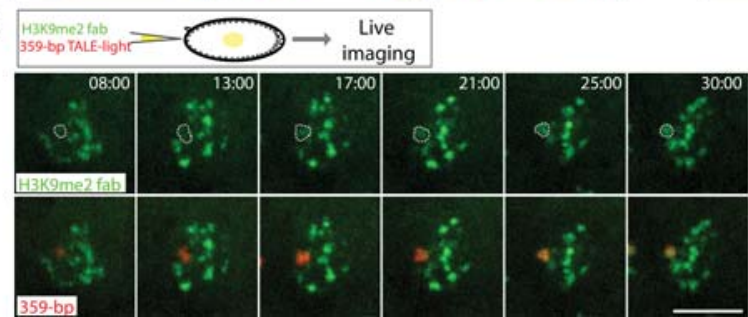

B

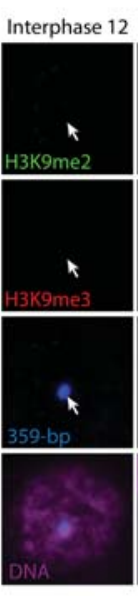

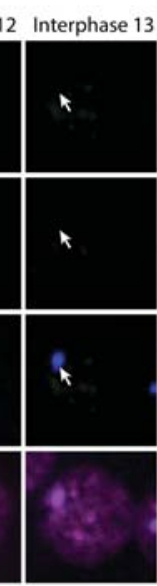

$4 \frac{\text { Interphase } 14}{4.5 \mathrm{~min}-29.5 \mathrm{~min}}$

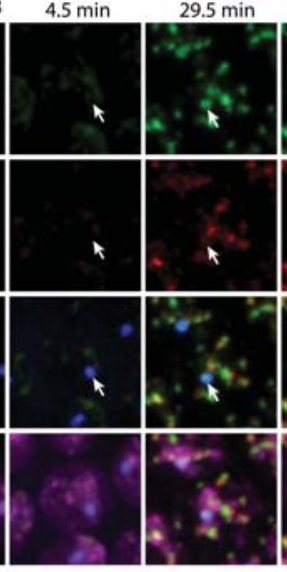

D $42.0 \mathrm{~min}$ into interphase 14

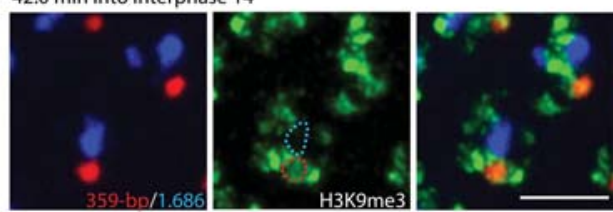

$\mathbf{F}$

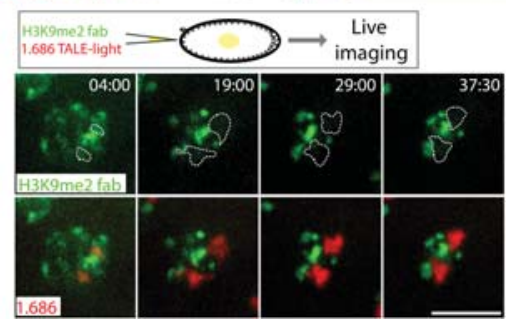

Figure 2. Differential accumulation of methylated H3K9 on the 1.686 and 359-bp repeats. $(A)$ Schematic of the in vivo and in vitro applications of the TALE-lights. (B) The appearance of H3K9me2 and H3K9me3 marks on the 359-bp repetitive sequence during embryogenesis. Fixed embryos at different developmental stages were stained with antibodies and TALE-light targeting the 359-bp repeat. $\mathrm{H} 3 \mathrm{~K} 9 \mathrm{me} 2$ is shown in green, H3K9me3 is shown in red, TALE-light staining of the 359-bp repeat is shown in blue, and DNA is shown in magenta. Arrows point to the 359-bp locus. All of the images were captured and processed using the same microscopic settings so that the fluorescent intensities faithfully reflect the relative quantities of the indicated modifications. Bar, $5 \mu \mathrm{m}$. $(C, D)$ Both $\mathrm{H} 3 \mathrm{~K} 9 \mathrm{me} 2$ $(C)$ and H3K9me3 $(D)$ accumulate on the 359-bp repeat (red dotted circles) but not on the 1.686 repeat (blue dotted circles) in cycle 14. Fixed interphase 14 embryos were stained with antibodies and TALE-lights. H3K9me2 or H3K9me3 is shown in green, and TALElight-stained 359-bp and 1.686 repeats are shown in red and blue, respectively. Bars, $5 \mu \mathrm{m} .(E, F)$ In vivo live imaging of accumulation of the H3K9me2 mark on particular repetitive sequences by combining the TALE-light imaging with the Fab-based live endogenous modification (FabLEM) technique (see the Materials and Methods). Time-lapse images of nuclei in the injected interphase 14 embryos are shown. The reformation of the interphase nucleus is set to be 00:00 (minutes:seconds). The Fab fragment recognizing the H3K9me2 mark is shown in green, and TALE-lights are in red. The dotted circles in the top panels outline the TALE-light-labeled regions. Bars, 5 um.

repetitive regions contained nucleosomes in the early embryo (Supplemental Fig. S2C,D), although the AT-rich 1.686 region seemed to either be less compact or have lower nucleosome occupancy (Krassovsky and Henikoff 2014) when compared with adjacent regions. Early embryos showed little or no staining for most of the histone modifications tested in these two repetitive regions, except for trace staining of acetylated H3K27 and H4 at the 359-bp loci early on (Supplemental Fig. S2F-M). In interphase 14, both H3K9me2 and H3K9me3 started to accumulate on the 359-bp repetitive sequences and were maintained in the following cell cycles (Fig. 2B). In contrast, no staining for H3K9 methylation was observed in the 1.686 region in interphase 14 (Fig. 2C,D), but staining was detected later in embryogenesis (Supplemental Fig. S2E, stage 11).

A Fab-based live endogenous modification (FabLEM) labeling technique has been developed to visualize histone modifications in vivo (Hayashi-Takanaka et al. 2011; Stasevich et al. 2014). To confirm the differential emergence of H3K9 methylation at 359-bp and 1.686 loci in developing embryos, we injected the TALE-light to visualize the corresponding satellite sequences and a FabLEM probe to label $\mathrm{H} 3 \mathrm{~K} 9 \mathrm{me}$. In agreement with the TALE-light staining results, the 359-bp region, but not the 1.686 region, gradually recruited the $\mathrm{H} 3 \mathrm{~K} 9 \mathrm{me} 2$ probe in interphase 14 (Fig. 2E,F). We conclude that the onset of significant accumulation of $\mathrm{H} 3 \mathrm{~K} 9 \mathrm{me} 2 / 3$ is delayed until 
cycle 14 and that it accumulates progressively following distinct time courses at different loci.

\section{Developmental regulation of heterochromatin formation on the 359-bp repeat}

The differential accumulation of $\mathrm{H} 3 \mathrm{~K} 9 \mathrm{me} 2 / 3$ during cycle 14 suggests heterochromatin formation on the 359-bp repeat but not on the 1.686. It is thought that $\mathrm{H} 3 \mathrm{~K} 9 \mathrm{me} 2 / 3$ binds and recruits HP1a, and the establishment of a stable HP1a-bound state underlies or at least marks the molecular process of heterochromatin formation.

To characterize how the 359-bp repeat is heterochromatinized, we made recombinant HP1a protein with a GFP tag fused to its $\mathrm{N}$ termini (Supplemental Fig. S3A). Localization of the GFP-tagged HP1a to spreads of the polytene chromosomes of the salivary gland resembled that described for the endogenous HP1a, and selective localization in early embryogenesis was seen with endogenous as well as tagged HP1a (Supplemental Fig. S3D-F). We injected the GFP-HP1a along with the TALE-light probe to visualize the HPla recruitment step in heterochromatin formation on particular satellite sequences in developing embryos. Western blot analysis showed that the injected exogenous GFP-HPla was at a concentration comparable with that of the endogenous HP1a (Supplemental Fig. S3B).

At each mitosis, the TALE-light probe targeting the 359-bp repeat was displaced from mitotic chromosomes but soon reaccumulated to the 359-bp loci as the nuclei exited mitosis. We followed the accumulation of GFP-HP1a within the TALE-light-labeled region in each interphase during early embryogenesis (e.g., Fig. 3B, bottom panels, dotted circle) and quantified the enrichment of GFP-HP1a at the 359-bp loci by calculating the fold enrichment over a control area in the same nucleus (Materials and Methods; Supplemental Fig. S3C). From interphase 11 to interphase 13, GFP-HPla was enriched at several discrete loci in the nucleus, but little was found in the 359-bp region (Fig. 3A; Supplemental Movie S2), which was consistent with the observation that H3K9me2/3 was absent at this stage. Dramatic accumulation of GFP-HPla at the 359-bp loci was observed in interphase 14 (Fig. 3B; Supplemental Movie S3). After a rapid accumulation phase from $15 \mathrm{~min}$ to $30 \mathrm{~min}$ into interphase 14, the amount of GFP-HP1 within the 359-bp region reached a plateau (Fig. 3E, blue curve). This HP1abound state of the 359-bp repeat was stably maintained thereafter (Fig. 3F, blue curve), except for transient mitotic displacement. As shown in Figure 3C, in interphase 15, the 359-bp loci was decorated by GFP-HP1a right after mitotic exit and became part of the chromocenter.

It is noteworthy that we observed a small amount of GFP-HP1a within the 359-bp region at the end of interphase 13 and even interphase 12 (Fig. 3A; Supplemental Movie S2), suggesting that the rapid cell divisions interrupted recruitment to the 359-bp repeat. To test this, we knocked down all three mitotic cyclins by RNAi to arrest the embryos in interphase 13 (Farrell et al. 2012) and ana- lyzed the accumulation of GFP-HP1a at the 359-bp loci. Indeed, stopping the rapid cell cycle allowed early recruitment of GFP-HPla to the 359-bp loci (Fig. 3G, orange curve). This result suggests that interphase extension at the MBT is sufficient for the formation of heterochromatin at the 359-bp loci.

The 1.686 repeat, on the contrary, did not recruit GFPHP1a during interphase 14 (Fig. 3D-F). These observations, based on GFP-HP1a, were confirmed by antibody staining of endogenous HP1a (Supplemental Fig. S3D). Together, our results reveal clear distinctions in the process of heterochromatin formation between different repetitive sequences. As building blocks of the constitutive heterochromatin, it is apparent that different repetitive sequences take different routes to reach the heterochromatic state during embryogenesis.

\section{Ordering the events during heterochromatinization}

The heterochromatinization of the 359-bp repeat in interphase 14 provides an opportunity to dissect the underlying molecular mechanism. It is known that $\mathrm{H} 3 \mathrm{~K} 9 \mathrm{me} 2 / 3$ helps recruit HP1a, and HP1a promotes the spreading of the H3K9me2/3 mark by recruiting histone methyltransferase (Canzio et al. 2014). We thus tested what initiates the heterochromatinization process on the 359-bp repeat.

The N-terminal chromodomain $(\mathrm{CD})$ of HP1a specifically recognizes the $\mathrm{H} 3 \mathrm{~K} 9 \mathrm{me} 2 / 3$ mark, whereas the C-terminal chromoshadow domain (CSD) dimerizes and forms an interface that recruits proteins containing the PxVxL motif, where $\mathrm{x}$ is any amino acid (Supplemental Fig. S4B,C). A single amino acid substitution in the CD (V26M) abolishes the recognition of $\mathrm{H} 3 \mathrm{~K} 9 \mathrm{me} 2 / 3$, and a substitution (W200A) in the C-terminal extension sequence disrupts the binding of the $\mathrm{PxVxL}$ motif without affecting HP1a's dimerization (Mendez et al. 2011, 2013; Canzio et al. 2014). The V26M and W200A double mutant lost all of the specific localization in interphase nuclei (Fig. 4A; Supplemental Fig. S4A), suggesting that one or the other targeted site is required for HPla binding at this developmental stage. Interestingly, restoration of a functional $\mathrm{CD}$ and hence the $\mathrm{H} 3 \mathrm{~K} 9 \mathrm{me} / 3$ binding activity (HP1a-W200A) brought back a subset of the HPla foci but did not rescue the timely accumulation at the 359-bp locus (Supplemental Movie S4). It was only faintly enriched within the 359-bp region toward the end of interphase 14 (Fig. 4B), and quantification analysis showed that it lacked the rapid accumulation phase seen with the wild type (Fig. 4E). In contrast, HP1a-V26M, which is capable of binding to the PxVxL motif but not the H3K9me2/3 mark, was efficiently recruited to the 359-bp loci (Fig. 4C; Supplemental Movie S5), although the quantification analysis indicated that its accumulation curve plateaued at a reduced height (Fig. 4E), which could simply be due to the increased background or might imply that a deficiency in H3K9me2/3 recognition compromises the late accumulation phase of HP1a. Together, these results suggested that the initial recruitment of HP1a to the 359-bp repeat did not depend on the capability to bind the $\mathrm{H} 3 \mathrm{~K} 9 \mathrm{me} 2 / 3$ 
A

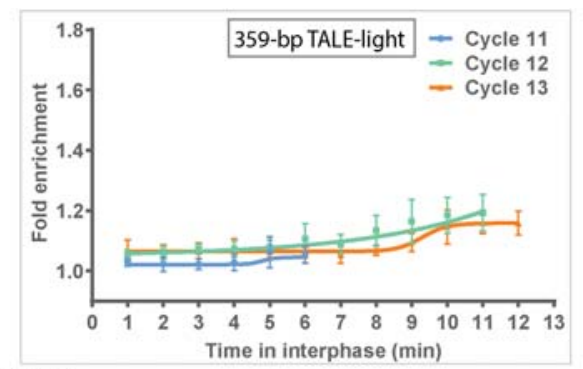

E

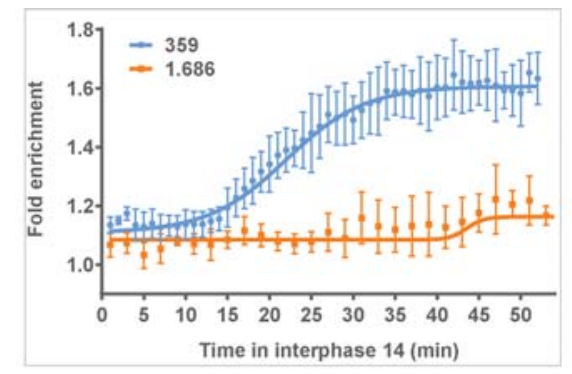

B Interphase 14

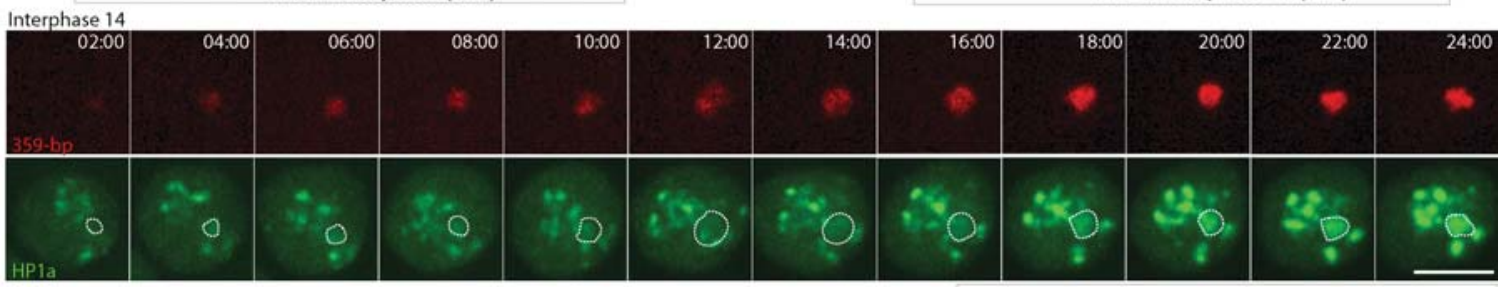

C

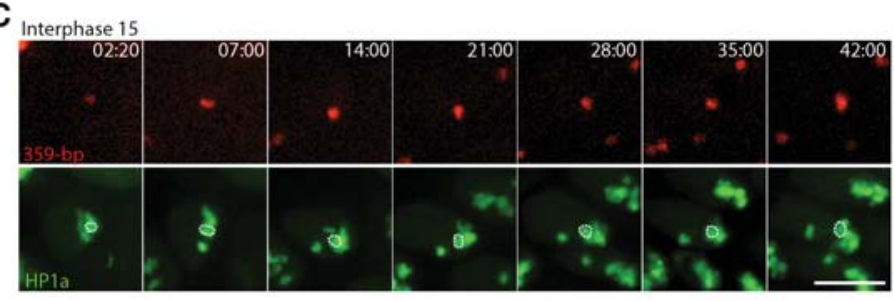

F

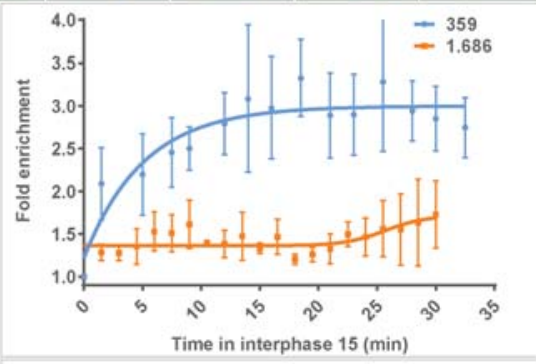

D Interphase 14

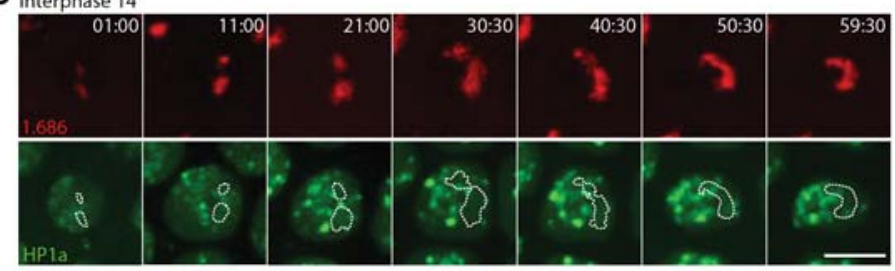

G

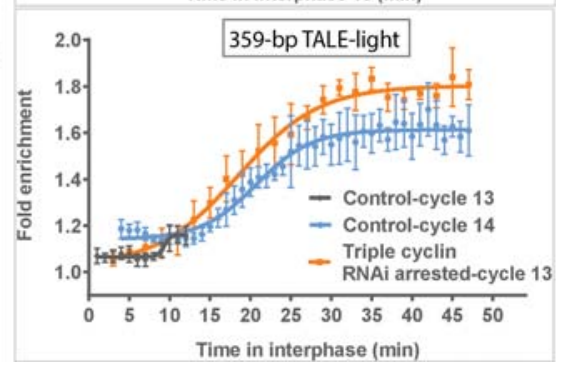

Figure 3. Developmentally regulated heterochromatin formation on the 359-bp repeat. (A) Quantification of GFP-HP1a accumulation at the 359-bp loci from interphase 11 to interphase 13 (for detailed method, see the Materials and Methods; Supplemental Fig. S3C). $n>3$. Error bars represent the SD. $(B, C)$ Frames from videos at the indicated times (minutes:seconds) showing GFP-HP1a accumulation at the 359-bp loci (dotted circle) in interphase $14(B)$ and interphase $15(C)$. Note that, in interphase 15, GFP-HP1a is rapidly recruited to the 359bp region after mitosis. The reappearance of interphase nucleus is set to be 00:00. Bars, $5 \mu \mathrm{m}$. $(D)$ Time-lapse images showing no obvious accumulation of GFP-HP1a at the 1.686 loci in interphase 14. Bar, $5 \mu \mathrm{m}$. $(E, F)$ Quantification of GFP-HP1a accumulation at the 359-bp (blue curve) and 1.686 (orange curve) loci in interphase $14(E)$ and interphase $15(F) . n>3$. Error bars represent the SD. (G) GFP-HP1a accumulation at the 359-bp loci in triple-cyclin RNAi-arrested interphase 13 embryos (orange curve) is comparable with that in the control interphase 14 embryos (blue curve). GFP-HPla accumulation in control interphase 13 is also shown for comparison (gray curve). $n>3$. Error bars represent the SD.

mark but required an ability to interact with proteins that contain the PxVxL motif. Consistent with this, the HP1aI191E mutant that cannot dimerize and hence loses the ability to bind the PxVxL ligand (Brasher et al. 2000; Mendez et al. 2011, 2013; Canzio et al. 2014) also failed to accumulate on the 359-bp repeat (Supplemental Fig. S4D).

A small amount of GFP-HP1a could be seen in the 359bp region at the end of interphase 13 (Supplemental Fig. S4A,C, first two columns). We further quantified and compared this premature recruitment among different HP1a mutants. As shown in Figure 4D, no significant reduction was observed for HP1a-V26M when compared with the wild type; however, mutants without the ability to bind the PxVxL motif failed to be recruited at this stage. Thus, we conclude that the initial recruitment of HP1a to the 359-bp region does not depend on binding to $\mathrm{H} 3 \mathrm{~K} 9 \mathrm{me} 2 / 3$. This is consistent with the late and gradual accumulation of these methyl marks described above.

\section{Altering the position of the 359-bp repeat within the nucleus does not alter the timing of HP1 a recruitment}

We wanted to know what signal guided the HP1a accumulation on the 359-bp repeat. Constitutive heterochromatin is spatially separated from euchromatin in the nucleus of most somatic cells and is often positioned 


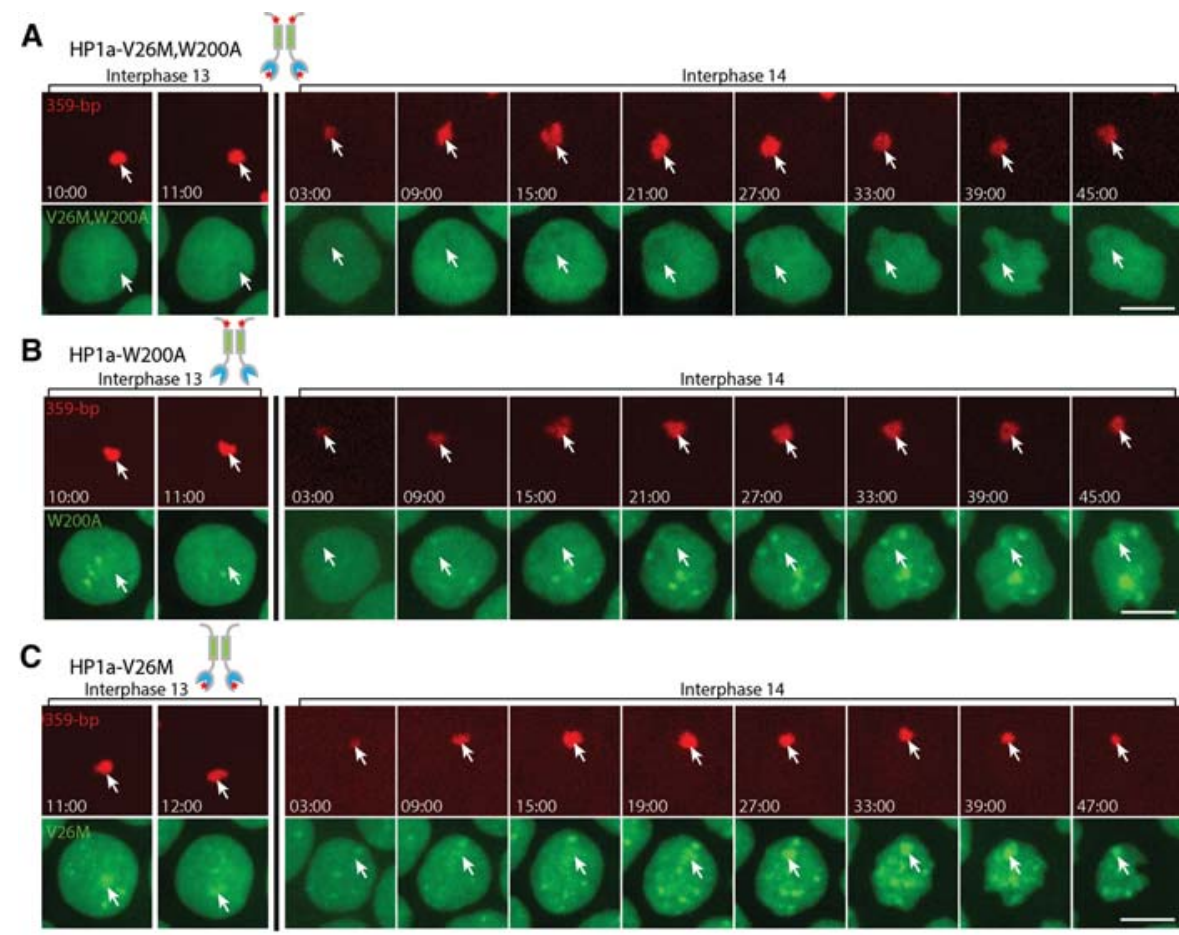

D

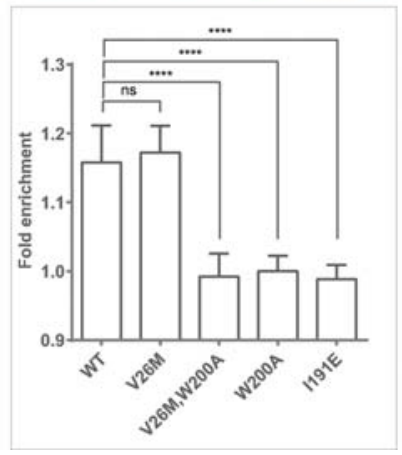

E

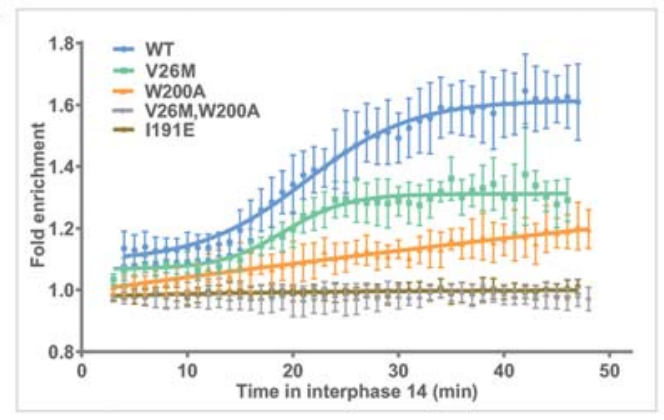

Figure 4. Recruitment of HP1a to the 359-bp repeat does not require the CD. $(A-C)$ Time-lapse images showing accumulation of the indicated GFP-HP1a mutant on the 359-bp repeat at the end of interphase 13 (first two columns) or during interphase 14 (the rest of the columns). Note that the reformation of the nucleus in each interphase is set to be 00:00 (minutes:seconds). Red stars in the schematic of HP1a structure indicate the position of the point mutations. Arrows point to the corresponding 359-bp loci. Bars, $5 \mu \mathrm{m}$. $(D)$ Enrichment of the indicated GFP-HP1a protein within the 359-bp region at the end of interphase 13. The V26M mutant is enriched at the 359-bp loci similar to the wild-type HP1a (unpaired $t$-test, $P=0.5546$ ), whereas the W200A, V26M/W200A, and I191E mutants are not enriched (unpaired $t$-test, $P<0.0001)$. Error bars represent the SD. (E) Quantification of different GFP-HP1a mutants accumulating at the 359-bp loci in interphase 14. Error bars represent the SD. $n>5$.

adjacent to the nuclear lamina and the periphery of the nucleoli (Padeken and Heun 2014). The unique nuclear position has been suggested to play a role during the establishment of heterochromatin (Jachowicz et al. 2013). We assessed the functional input of nuclear position during the heterochromatinization of the 359-bp repeat.

The majority of the 359-bp repeat localizes on the proximal end of the X chromosome. In the scute 8 mutant, which is associated with a major inversion on the $\mathrm{X}$ chromosome with an end point within the proximal part of the 359-bp repeat, most of the 359-bp repetitive sequences are translocated to the distal end of the X chromosome, with a small block of the 359-bp repeat remaining at its original centromere-proximal position (Supplemental Fig. S5C,D).
We characterized the GFP-HP1a accumulation at the large, more telomeric block of the 359-bp repeat, which is positioned at the basal pole of the nuclei (Supplemental Fig. S5F), as well as the smaller, more centromeric block of the repeat, which is positioned apically (Supplemental Fig. S5E). GFP-HP1a was recruited to both loci of the 359 -bp repeat simultaneously in interphase 14 , suggesting that nuclear position at this stage is not a major contributor to the formation of heterochromatin. Quantification indicated that the accumulation of GFP-HP1a to the basal 359-bp locus plateaued at a reduced height (Supplemental Fig. S5G), which might be partially due to the measurement inaccuracies during live embryo imaging caused by the elongating nuclei in interphase 14 (Waters 2009), 
which pushed the basal 359-bp locus up to $14 \mu \mathrm{m}$ away from the coverslip.

\section{Maternal cues guide heterochromatin formation}

A widespread feature of early embryogenesis is that the mother preloads the egg with material that directs most of the early developmental programs (Farrell and O'Farrell 2014). We reasoned that maternal signals might guide the heterochromatin formation on the 359-bp repeat. To test this, we removed the 359-bp repeat from the mother's genome and evaluated the heterochromatinization of the 359-bp repeat in the offspring embryos.
Repetitive sequences evolve rapidly. Another closely related species, Drosophila simulans, has no 359-bp or 359-bp-like repeat in its genome and can be crossed to D. melanogaster (Ferree and Barbash 2009). As illustrated in Figure 5A, when a female $D$. simulans was husbanded with a male $D$. melanogaster, the $F 1$ hybrid female embryos would have the maternal supply from the $D$. simulans mother that lacks the 359-bp repeat and would meanwhile obtain a copy of the 359-bp repeat from the $D$. melanogaster father. Most of these hybrid female embryos exhibited an early embryonic lethality due to chromosome missegregation in the syncytial cycles (Supplemental Fig. S5A,B). However, a small fraction developed to a later
A

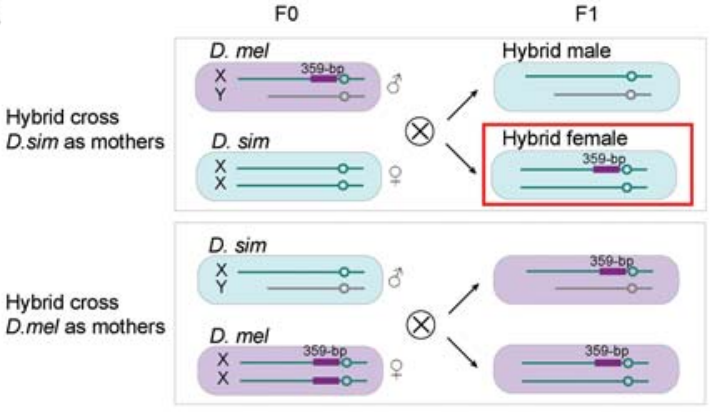

B

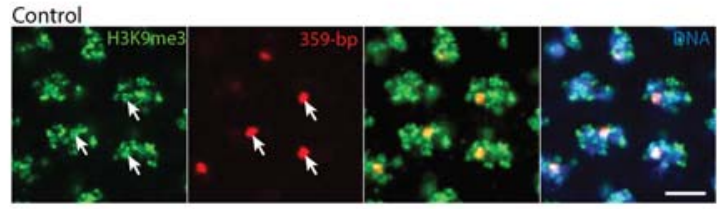

C Hybrid female

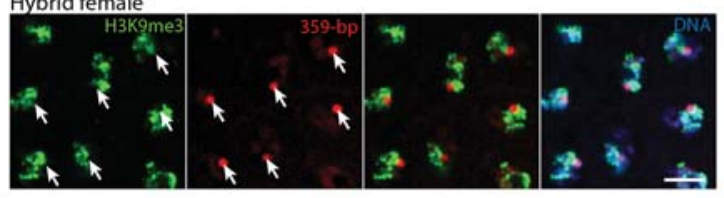

D $\begin{aligned} & \text { GFP-HPla } \\ & \text { 359-bp TALE-light }\end{aligned} \begin{gathered}\text { Hybrid } \\ \text { femaleme }\end{gathered} \longrightarrow \begin{gathered}\text { Live } \\ \text { imaging }\end{gathered}$
E

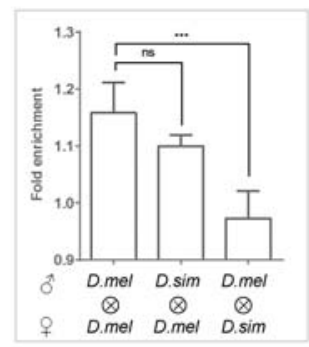

$\mathbf{F}$

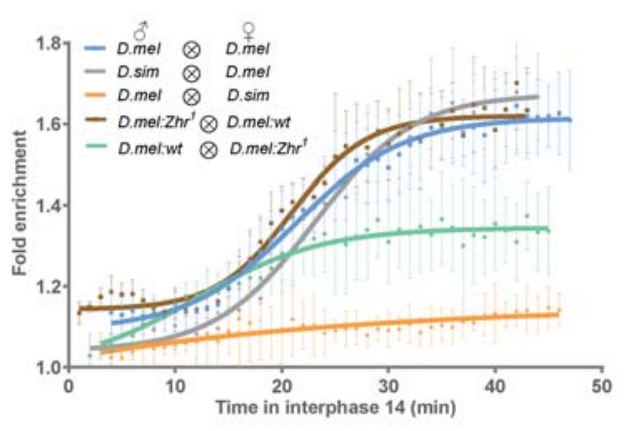

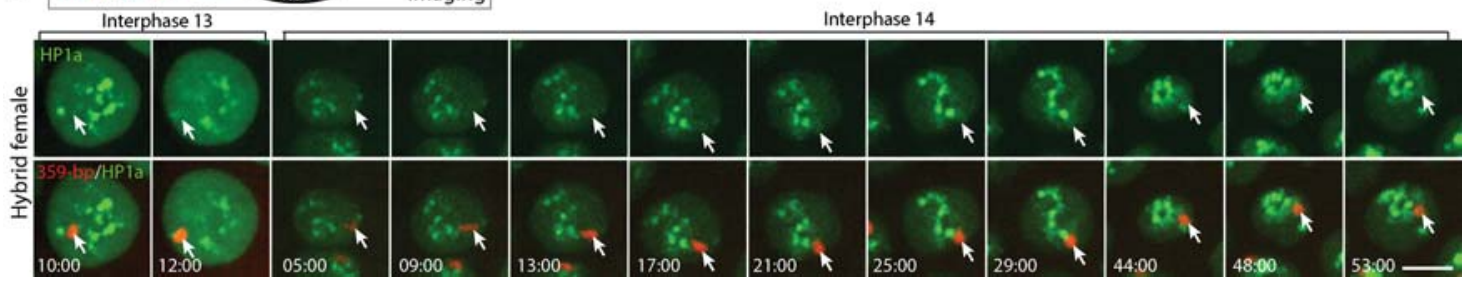

Figure 5. Maternal cues guide heterochromatinization of the 359-bp repeat. (A) Schematic of the hybrid cross between $D$. simulans (D. sim) and D. melanogaster (D. mel). Only sex chromosomes are shown. Note that the 359-bp repeat located on the X chromosomes of $D$. melanogaster is absent in $D$. simulans. $(B, C)$ TALE-light and H3K9me3 antibody stainings in the control and hybrid female embryos produced from the $D$. simulans and $D$. melanogaster cross. The H3K9me3 histone mark (arrows) exists within the 359-bp region in the control $(B)$ but not the hybrid $(C)$ female embryos. H3K9me3 is shown in green, the 359-bp repeat is shown in red, and DNA is shown in blue. Arrows point to the corresponding 359-bp loci. Bars, $5 \mu \mathrm{m}$. (D) Frames from videos at the indicated times (minutes:seconds) show a lack of GFP-HP1a accumulation on the 359-bp repeat (arrowheads) in the hybrid female embryos. Note that the reappearance of the nucleus in each interphase is set to be 00:00. Arrows point to the corresponding 359-bp loci. Bar, $5 \mu \mathrm{m}$. (E) Enrichment of GFP-HP1a within the 359-bp region at the end of interphase 13 in 359-bp repeat-bearing embryos produced from the indicated crosses. No significant difference was observed in embryos laid by $D$. melanogaster mothers (unpaired $t$-test, $P=0.0653$ ); however, embryos from the $D$. simulans mothers have less GFP-HP1a at the 359-bp loci when compared with control (unpaired $t$-test, $P=0.0002)$. Error bars represent the SD. (F) Quantification of GFP-HP1 a accumulation in interphase 14 at the 359-bp loci in the 359-bp-positive embryos produced from the indicated crosses. Note that the $Z \mathrm{hr}^{1}$ mutant of $D$. melanogaster $\left(D\right.$. mel:Zhr $\left.{ }^{1}\right)$ lacks the 359-bp repeat on the X chromosome but still has many tiny 359-bp derivatives on the autosomes. $n>5$. Error bars represent the SD. 
embryonic stage beyond the MBT. We analyzed the H3K9 methylation in these embryos. While H3K9me3 was found on the 359-bp repeat in control interphase $14 \mathrm{em}$ bryos (Fig. 5B, arrow), it was absent in the hybrid female embryos (Fig. 5C, arrowheads). Consistently, in these embryos, the HP1a accumulation at the 359-bp locus was greatly reduced when compared with that in the control or the embryos from the reciprocal cross (Fig. 5D,F; Supplemental Movie S6). The initial recruitment of HP1a at the end of interphase 13 was also compromised in these embryos. In the reciprocal cross in which $D$. melanogaster was the mother, embryos exhibited early recruitment of HP1a, whereas embryos from the $D$. simulans mother failed to do so (Fig. 5E).

The cross-species mating suggests that the maternal signal might depend on the maternal presence of the 359-bp repeat. We wanted to test this with $D$. melanogaster strains. Besides a major block of 359-bp repeat on the $\mathrm{X}$ chromosome, D. melanogaster has many tiny 359-bp derivatives on its autosomes (Wei et al. 2014). The $Z h r^{1}$ mutant lacks the majority of the 359-bp repeat due to compound X chromosomes but still has the autosomal 359-bp derivatives (Ferree and Barbash 2009). We mated the $Z h r^{1}$ females with control males and analyzed HP1 a accumulation to the paternally derived 359-bp locus in the offspring female embryos. The removal of most of the 359-bp repeat from the maternal genome had a quantitative effect, as the accumulation of HP1a in interphase 14 at the 359-bp locus was significantly reduced in the offspring embryos when compared with that in the control (Fig. 5F; Supplemental Movie S7). In contrast, in the reciprocal cross in which the normal 359-bp repeat was present in the maternal parent, progeny embryos showed normal accumulation of HP1a, indicating that the effect of the deletion on HP1a recruitment is a maternal effect phenotype (Fig. 5F).

We conclude that maternally provided factors contribute to the heterochromatinization of the 359-bp repeat. This maternal contribution appears to depend on the maternal presence of the 359-bp sequences. Deficits of maternal 359-bp sequences specifically affect the 359-bp locus in the progeny embryos.

Establishment of stable HP1a binding delays the timing of replication in $S$ phase

The observed selective heterochromatinization of the 359-bp repeat in interphase 14 could potentially explain the previously reported sudden delay in replication timing of this repeat in interphase 15 (Yuan et al. 2014). To directly test whether the establishment of the HP1abound state delays replication, we induced HP1a accumulation on the 1.686 repeat and measured its timing of replication. Artificial tethering $\mathrm{HP} 1 \mathrm{a}$ to DNA has been shown to induce local heterochromatin formation (Hathaway et al. 2012). We fused HPla to the $C$ terminus of the TALE-light probe recognizing the 1.686 repeat and injected a low level of this 1.686-HP1a fusion protein into the embryo to induce ectopic heterochromatin formation (Fig. 6A,B). The targeted HPla fusion protein induced ro- bust accumulation of untargeted GFP-HP1a at the 1.686 loci in interphase 15 (Fig. 6D). Moreover, the replicationcoupled decompaction of the 1.686 loci seen in the control embryos was postponed (Fig. 6C,D), indicating a delay in replication. We injected GFP-PCNA to directly visualize the bulk replication of the 1.686 repeat and compared its timing in embryos with or without the 1.686-HP1a. Indeed, the presence of the 1.686-HP1a fusion protein delayed the replication of the 1.686 in interphase 15 (Fig. 6E; Supplemental Fig. S6A).

To examine the consequences of loss of HPla recruitment, we used the GFP-PCNA reporter to follow the replication timing of the $359-$ bp repeat in the $D$. simulans and $D$. melanogaster hybrid embryos in which the 359-bp repeat failed to recruit HP1a. The replication of the 359-bp locus was advanced when compared with that in the control embryos (Fig. 6F; Supplemental Fig. S6B). These two sets of experiments provided direct evidence for the idea that heterochromatin formation delays the local timing of replication in S phase. We thus conclude that the sudden delay of replication timing of the 359-bp repeat in interphase 15 is due to the developmentally regulated heterochromatinization of this repeat.

As summarized in Figure 6G, during the syncytial blastoderm stage, high Cdk1 activity and/or the rapid cell cycle inhibit the accumulation of HP1a at the 359-bp loci. At the MBT in interphase 14, down-regulation of Cdk1 (Farrell and O'Farrell 2014) and other developmental inputs (Blythe and Wieschaus 2015) slows DNA replication and extends interphase, allowing the HP1a accumulation and hence heterochromatin formation on the 359-bp repeat. This local change in chromatin landscape impacts the DNA replication schedule in the following cell cycles, as the heterochromatic state of the 359-bp repeat selectively delays its timing of replication relative to the other repeats.

\section{Discussion}

TALE-lights: visualizing specific DNA sequences in vivo and in vitro

New tools are needed to probe the complex and dynamic nature of the organization of the eukaryotic genome. For $>30$ years, FISH has been the dominant method to label a given DNA sequence (Levsky and Singer 2003), but the requirement of denaturation of DNA often compromises the integrity of the sample and hinders its applications in living organisms. The integration of the lacO or TetO repeats into a particular genomic locus and the use of fluorescently labeled LacI or TetR protein provide strategies to visualize DNA targets live (Robinett et al. 1996). However, these methods lack the flexibility in target selection. Recent breakthroughs have allowed the systematic engineering of DNA sequence recognition, which stimulated the development of several new DNA visualization methods, including techniques based on the zinc fingers (ZFs) (Lindhout et al. 2007), the CRISPR/dCas9 (Chen et al. 2013; Anton et al. 2014; Deng et al. 2015), and the TALEs (Ma et al. 2013; Miyanari et al. 2013; Thanisch 
A

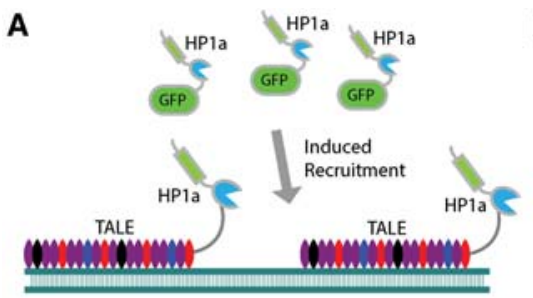

C Control +1.686 -mCherry Interphase 15

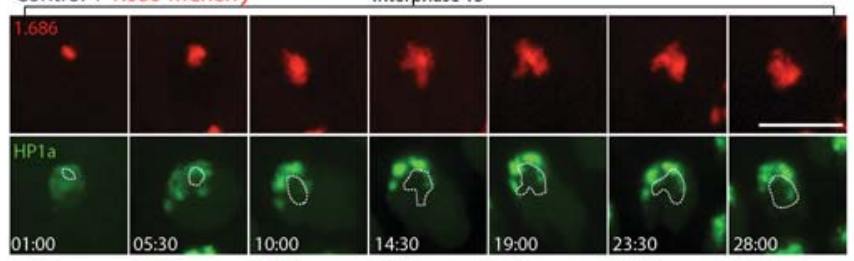

D $1.686-\mathrm{HP} 1 \mathrm{a}+1.686-\mathrm{mCherry}$

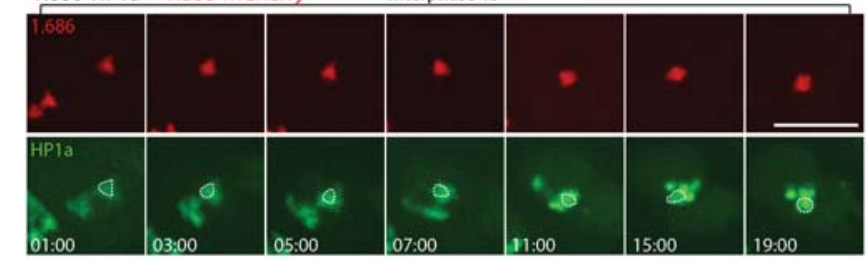

E

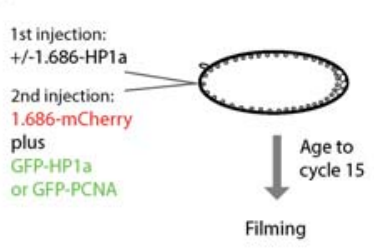

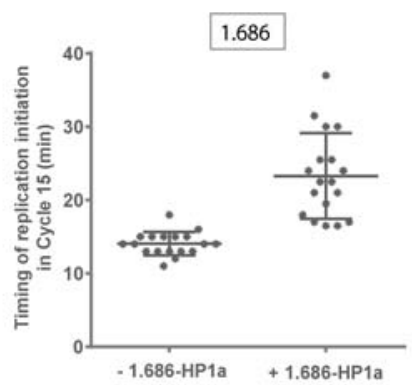

$\mathbf{F}$

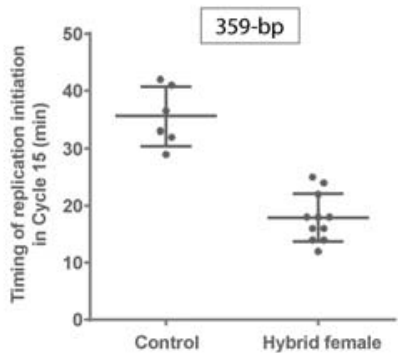

G Cycle13

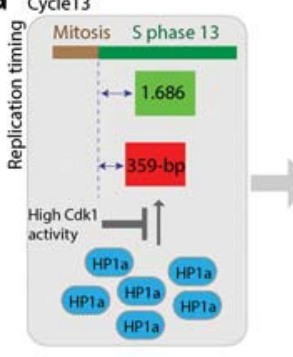

Cycle 14

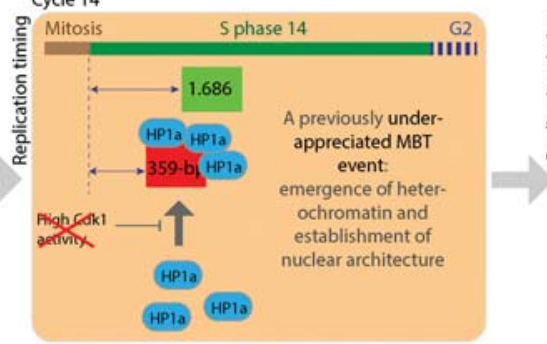

Cycle 15

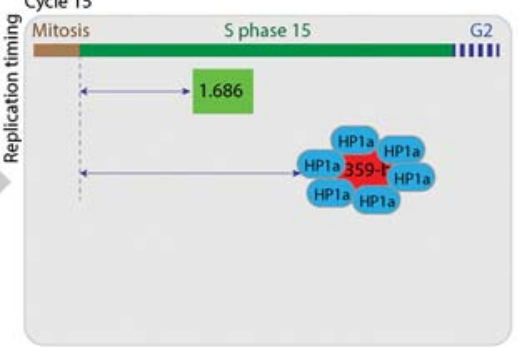

Figure 6. Establishment of the HP1a-bound state delays the timing of replication in S phase. $(A)$ A cartoon depicting the induced accumulation of GFP-HP1a by the TALE-HP1a fusion protein. $(B)$ Schematic of the experiment showing the injections. $(C, D)$ Time-lapse images show that GFP-HP1a is absent at the 1.686 locus (dotted circles in green panels) in control interphase 15 embryos $(C)$ but is recruited to the 1.686 region when the $1.686-\mathrm{HP} 1$ a fusion protein is present $(D)$. The reformation of the interphase 15 nucleus is set to be $00: 00$ (minutes:seconds). Bars, $5 \mu \mathrm{m}$. (E) Replication timing of the 1.686 repetitive sequence in interphase 15 with or without the injection of the 1.686-HP1a fusion protein. The induced HP1a accumulation on 1.686 significantly delays its timing of replication (unpaired $t$-test, $P<0.0001)$. Error bars represent the SD. $(F)$ Replication timing of the 359-bp repeat in interphase 15 . The 359-bp repeat in the hybrid female embryos from the $D$. simulans and $D$. melanogaster hybrid cross does not recruit HPla, and its timing of replication in interphase 15 is significantly earlier than that in the control embryos (unpaired $t$-test, $P<0.0001$ ). Error bars represent the SD. (G) A graphic summary depicting HP1a accumulation on the 359-bp repeat around the time of MBT and its influence on the replication timing.

et al. 2014; Yuan et al. 2014). Our TALE-lights belong to the last.

The sequence-specific DNA recognition by a TALElight comes from the one-to-one binding of the TALE modules to each of the DNA bases. Therefore, its programmability is as flexible as the CRISPR/dCas9 and better than the ZFs, as one ZF domain recognizes a 3-bp 5'GNN-3' DNA sequence (Segal et al. 1999). Moreover, our results demonstrate that the TALE-lights work well in both fixed and live conditions. Because of this feature, we believe that the TALE-lights will be a valuable tool for the studies of genome organization. At this stage, we visualized only repetitive DNA elements. By adapting proper signal amplification strategies, single-copy genes might become visible.
The use of exogenous proteins or RNPs to label endogenous DNA elements in live cells could potentially interfere with normal cellular functions. Indeed, it has been reported that the binding of LacI to the $1 a c O$ repeats blocks DNA replication (Duxin et al. 2014). The use of TALElights in our experiments, however, was seemingly compatible with DNA replication, affecting neither cell cycle length nor embryogenesis (Yuan et al. 2014). Since TALElights can be used in vitro as a "sequence-specific DNA antibody," we examined fixed embryos and found that the appearance of histone marks on repetitive sequences was consistent with the dynamics scored in live embryos. This again suggests that TALE-lights binding in vivo did not cause major disruptions. In addition, we noticed that the majority of the TALE-light was displaced from the 
mitotic chromosomes, whereas the GFP-LacI stayed on (Holt et al. 2008). We reason that TALE-lights have a weaker DNA-binding affinity and thus are better tolerated by the cells. Nevertheless, the results generated by such a method should always be interpreted with caution, and it is important to point out that we did observe chromosome missegregation when a much higher concentration of the TALE-light was injected into the embryo.

\section{Constitutive heterochromatin formation in development}

The ability of the TALE-lights to discriminate individual satellite sequences has given us the capacity to reveal their differences in the establishment of constitutive heterochromatin during early embryogenesis. The 359-bp repeat recruits HPla and becomes heterochromatinized in interphase 14, and it appears to typify one group of repeat sequences to which the initial recruitment of HP1a does not depend on the presence of $\mathrm{H} 3 \mathrm{~K} 9 \mathrm{me} 2 / 3$. This group of repeat sequences was visualized as distinct loci of accumulation of the mutant HP1a (V26M), which is incapable of binding to H3K9me2/3 (Fig. 4C). In contrast, the HP1a mutant with a deficient CSD (W200A) failed to localize to the 359-bp repeat but did localize to several foci in interphase 14 that appear to represent a second group of repeat sequences that recruits $\mathrm{HPla}$ using an alternative mode of interaction, perhaps by CD binding of $\mathrm{H} 3 \mathrm{~K} 9 \mathrm{me} 2 / 3$ (Fig. 4B). However, we have yet to identify a TALE-light marking this group of foci, and additional work is needed to characterize the mode of HP1a recruitment to these sites. The third group of repetitive sequences includes 1.686. In terms of the classical hallmarks of heterochromatin, these repeats remain largely naive at the time of the MBT. We do not know the molecular process of heterochromatin formation on these repeats.

While we use the term heterochromatinization to describe the ensemble of changes associated with recruitment of HP1a to the 359-bp satellite, our findings suggest complexity in the processes that establish heterochromatin and some resulting ambiguity in terminology. HP1a and the histone modification with which it associates, $\mathrm{H} 3 \mathrm{~K} 9 \mathrm{me} 2 / 3$, are often taken as markers that define heterochromatin. Even though this study provides direct evidence for the functional impact of a transition in chromatin structure marked by $\mathrm{HPla}$ recruitment, the results also highlight the diversity in forms of heterochromatin. The satellite sequences have distinctive features prior to the recruitment of HP1a, including compaction and late replication in cycle 14 (Shermoen et al. 2010). Although the delay in replication of the 359-bp repeat prior to HP1 a binding is slight, it is nonetheless delayed in cycle 14 . Furthermore, the 1.686 satellite fails to mature to an HP1a-bound form until later in development yet maintains a late replication program. These observations reinforce a perspective that many factors influence the formation of "heterochromatin," and the degree of uniformity of the resulting chromatin structures remains to be established.
Controlling heterochromatinization in time and space at the $M B T$

The whole block of the 359-bp repeat undergoes heterochromatinization precisely in interphase 14 . How does the embryo know when to form this heterochromatin? As mentioned earlier, fly embryos begin development with extremely fast nuclear divisions characterized by unusually high mitotic Cdk activity (Farrell and O'Farrell 2014). Given that the binding of HPla and its silencing activity are both regulated by dynamic phosphorylation/ dephosphorylation events (Zhao and Eissenberg 1999; Zhao et al. 2001), the high kinase activity and the rapid cell divisions in the early embryo might curb the establishment of the stable heterochromatic state. Indeed, our results showed early accumulation of HPla on the 359-bp repeat when Cdk1 activity was down-regulated and the cell cycle was arrested, suggesting that factors needed for heterochromatinization of the 359-bp repeat are already present in the early embryos and that developmentally regulated cell cycle slowing provides the first opportunity for their action (Fig. 6G). However, recruitment of HP1a to foci of repeated sequences does not occur immediately in cycle 14, and the precise timing of this recruitment suggests sophisticated and as yet unknown regulatory circuitry.

Notably, constitutive heterochromatin is not always heterochromatic, at least according to the molecular hallmarks of this state. What marks the 359-bp sequences for selective formation of heterochromatin? It is notable that some repeat sequences, such as those of the 359-bp locus, are selectively compacted during early mitotic cycles despite the absence of H3K9me2/3 (Shermoen et al. 2010), suggesting that some intrinsic feature of the satellite sequence might specify its special behavior. However, because only some of the constitutively heterochromatic repeats recruit $\mathrm{HP} 1 \mathrm{a}$ and because there have been numerous findings suggesting that maternal signals might act transgenerationally to direct aspects of gene activity, we sought to distinguish between a transacting maternal signal and sequence-autonomous features of the input.

Genetic manipulation of the amount of the 359-bp repeat in the mothers' genome (using either $D$. simulans or D. melanogaster $Z h^{1}$ mutants as mothers) (see Fig. 5) specifically influenced the heterochromatin formation on the 359-bp repeat in the offspring, whereas changes in the fathers' genome (the reciprocal crosses) had no such effect. These results suggest that maternal 359-bp sequences contribute to the zygotic establishment of heterochromatin at the 359-bp locus and encourage us to think of the type of signal that might be conveyed from mother to progeny. It has been established that particular RNAs expressed in the maternal germline are processed into a special class of small RNAs called piRNAs that act to silence gene expression. RNAs homologous to the 359-bp repeat were found in the germinal tissues, and the abundance of these transcripts was increased in mutants defective in components in the piRNA pathway, suggesting that transcription of these sequences occurs and that the piRNA pathway targets this expression (Usakin et al. 2007). Current 
understanding of piRNA suppression of the expression of repeated sequences suggests that copies of the sequence in a specialized piRNA cluster in heterochromatic regions are expressed, processed, and loaded onto an Argonaut protein called Piwi. Piwi in complex with a specific piRNA sequence appears capable of doing three things: directly targeting homologous DNA sequences to promote heterochromatin formation and suppress transcription, contributing to slicing activity that destroys the RNA products of homologous loci, and working in conjunction with other piRNA pathway components to amplify the piRNA signal by processing complementary and homologous RNA (Guzzardo et al. 2013). Piwi has been found to be associated with small RNAs derived from the 359-bp repeat region (Saito et al. 2006), consistent with a potential role of this pathway in regulating heterochromatin formation on these sequences. Our finding that HP1a is recruited to the 359-bp sequence at a specific time in cycle 14 and in a process that depends on the maternal presence of 359-bp sequences could be explained by suggestions that the 359-bp-derived piRNAs are required for timely heterochromatinization of these sequences, although the details of the mechanism remain to be addressed.

\section{Heterochromatin formation and the timing of replication}

We began with an interest in the developmental onset of late replication. We found that this onset is triggered by the down-regulation of mitotic cyclin:Cdk1, which, if active during interphase, can trigger early replication of otherwise late-replicating sequences (Farrell et al. 2012). While this finding indicates that a change in a "trans factor" acting on pre-existing heterochromatin might be responsible for this onset, we found that heterochromatic marks were introduced at about the time of onset of late replication (Shermoen et al. 2010). Additionally, we noted that the onset of late replication is modulated differently at different repeat sequences (Yuan et al. 2014). These later observations suggest that changes of the chromatin structure of the repeat regions (cis changes) also occur and might modulate the replication timing.

Widespread coupling between heterochromatin and late replication has fostered the idea that heterochromatin is late-replicating. Additionally, the genomic context has been shown to influence the timing of firing of origins of replication, a finding that is generally interpreted as showing that local chromatin structure specifies replication timing. Nonetheless, despite strong connections, experimental support for a causal connection between heterochromatin and replication timing is indirect. Global knockdown of HPla in Drosophila Kc cells by RNAi advanced the replication of centromeric repeats but delayed replication of many other genomic regions (Schwaiger et al. 2010). Such complex effects might be due to indirect effects of chromatin structure on overall genome arrangement (Sexton and Yaffe 2015).

By manipulating HP1a levels locally at specific repetitive loci, our results demonstrated that failure to establish a HPla-bound state at the 359-bp repeat locus advanced its replication timing in cycle 15 and that experimentally induced TALE-mediated HP1a recruitment to the 1.686 locus delayed its replication in cycle 15. This, together with the natural developmental program of HP1a recruitment to the different satellite sequences, strongly supports a causal connection between HP1a recruitment and replication timing. It should be noted, however, that recruitment of HP1a can engage a number of reinforcing interactions among factors promoting heterochromatin formation. Hence, the results argue for a causal connection, but not necessarily a direct or even simple connection, between HP1a recruitment and replication timing.

HP1a-positive regions unfold in conjunction with PCNA recruitment and replication and with recruitment of repair proteins and repair of DNA damage (Shermoen et al. 2010; Chiolo et al. 2011). It is presently unclear whether the events of repair and replication unfold the compacted heterochromatic structure or whether a separate decompaction process avails the compacted sequences of these reactions. However, in either case, compaction could impede these processes, and disassembly of the compacted structure might be regulatory. The activation and firing of replication origins involves complex assemblies regulated by several kinases and phosphatases. The dispersal of compacted heterochromatin might also be regulated by the dynamic phosphorylation/dephosphorylation events, and interplay between the structure and the activating processes might govern timing. More future studies are needed to fully uncover the underlying mechanisms of late replication, and we believe that the TALE-light technique will play an important role during this process.

\section{Materials and methods}

\section{TALE-light assembly}

Customized TALE arrays recognizing a given repetitive sequence were designed using TALE Nucleotide Targeter 2.0 (http://tale-nt.cac.cornell.edu). The following target sequences were chosen: 5'-AGCACTGGTAATTAGCTGCT-3' and 5'-AG CTGCTCAAAACAGATATT- ${ }^{\prime}$ to target the 359-bp repeat and 5'-AGAATAACATAGAATAACAT-3' to target the 1.686 repeat.

The TALE arrays were assembled using Golden Gate TALEN and TALE kit 2.0 (Addgene). Backbone plasmid MR015 was used in the final reaction. The full-length TALE array was then subcloned into the customized pET-28 bacterial expression vector carrying a C-terminal GFP or mCherry tag. These constructs were subsequently used to produce the recombinant TALE-light proteins.

\section{Fly stocks}

Drosophila strains were maintained on standard cornmeal-yeast medium. D. melanogaster strains used in this study were as follows: the Sevelen line as the wild type, scute 8 [full genotype is $\operatorname{In}(1) \mathrm{sc}^{8}, \mathrm{sc}^{8} \mathrm{y}^{31 \mathrm{~d}} \mathrm{w}^{\mathrm{a}}$; Bloomington Stock Center, no. 798], and $Z h r^{1}$ [full genotype is $X Y S . Y L, D f(1) Z h r$; provided by Dr. Daniel Barbash]. In the hybrid cross, $40-50 \mathrm{D}$. simulans virgins were 
mated to 50-60 Sevelen males in a confined vial for $2 \mathrm{~d}$. Flies from several such vials were pooled into a population cage for embryo collection.

\section{Protein production and microinjection}

TALE-lights as well as GFP-HP1a and GFP-PCNA were produced in BL21 (DE3)-competent Escherichia coli cells (Bioline). Briefly, BL21 (DE3) transformants cultured in Luria-Bertani medium were treated with $0.5 \mathrm{mM}$ IPTG for $12 \mathrm{~h}$ at room temperature to induce protein expression. Bacteria were pelleted by centrifugation and resuspended in lysis buffer (20 mM Tris at $\mathrm{pH} 7.9,500$ $\mathrm{mM} \mathrm{NaCl}, 10 \mathrm{mM}$ imidazole). Next, the bacteria were incubated for $1 \mathrm{~h}$ on ice in the presence of $0.1 \mathrm{mg} / \mathrm{mL}$ lysozyme and $10 \mu \mathrm{M}$ PMSF and further lysed by sonication (ultrasonic liquid processors; Misonix). The recombinant protein in the cleared bacterial lysate was purified using Ni-NTA agarose (Macherey-Nagel). After thorough washes with wash buffer $(20 \mathrm{mM}$ Tris at $\mathrm{pH}$ $7.9,500 \mathrm{mM} \mathrm{NaCl}, 20 \mathrm{mM}$ imidazole), the protein was eluted in elution buffer $(20 \mathrm{mM}$ Tris at $\mathrm{pH} 7.9,200 \mathrm{mM} \mathrm{NaCl}, 300$ $\mathrm{mM}$ imidazole) and then dialyzed into $40 \mathrm{mM}$ Hepes (pH 7.4) and $150 \mathrm{mM} \mathrm{KCl}$.

The microinjection was performed as previously described (Farrell et al. 2012). The TALE-lights were used at $1 \mu \mathrm{g} / \mu \mathrm{L}$, GFP-HP1a was used at $6 \mu \mathrm{g} / \mu \mathrm{L}$, and GFP-PCNA was used at $2 \mu \mathrm{g} / \mu \mathrm{L}$. The fusion protein $1.686-\mathrm{HP} 1 \mathrm{a}$ was used at $0.1 \mu \mathrm{g} / \mu \mathrm{L}$ to induce ectopic HP1a recruitment.

\section{Immunofluorescence and TALE-light staining}

Embryos were collected on grape agar plates, dechorionated for $2 \mathrm{~min}$ in $50 \%$ bleach, and fixed in methanol-heptane (1:1) for $5 \mathrm{~min}$. The fixed embryos were stored in methanol at $-20^{\circ} \mathrm{C}$. Before immunostaining, the embryos were first rehydrated gradually $(5$ min each in 1:3, 1:1, and 3:1 PTA:methanol and then 10 min in PTA). PTA consisted of PBS supplemented with $0.1 \%$ Triton $\mathrm{X}-100$, and $0.02 \%$ azide. The embryos were then blocked in PBTA (PTA plus 1\% BSA) for 30 min and incubated with primary antibodies $(1: 100$ in PBTA) for $1 \mathrm{~h}$ at room temperature or overnight at $4^{\circ} \mathrm{C}$. The following primary antibodies were used: anti-Histone 3 (Abcam, ab1791), anti-H3K9me1 (Active Motif, 39249), anti-H3K9me2 (Active Motif, 39683 and 39375), antiH3K9me3 (Active Motif, 39765; Millipore, clone CMA308), anti-H3K9ac (Abcam, ab10812), anti-H3K27ac (Abcam, ab4729), anti-H3K27me3 (Cell Signaling, 9733), and anti-H4ac (Millipore, 06-598). The embryos were washed three times for 5 min each in PBTA and incubated with the appropriate fluorescently labeled secondary antibodies (Molecular Probes) for $1 \mathrm{~h}$ in the dark at room temperature. They were then washed four times for $5 \mathrm{~min}$ each in PBTA and mounted in VectaShield mounting medium with DAPI (Vector Laboratories). For TALE-light stainings, GFP- or mCherry-tagged purified TALE-light protein (1:500) was included during the incubation with secondary antibodies.

\section{FabLEM imaging}

All the FabLEM reagents were kindly provided by Dr. Hiroshi Kimura. Briefly, histone modification-specific monoclonal antibodies were treated by protease digestion to generate Fab fragments, and the Fab fragments were then labeled with aminereactive fluorescent dyes to make the FabLEM probes (HayashiTakanaka et al. 2011). Under many experimental conditions, the FabLEM did not disturb normal cellular functions, including the HP1a binding in our experiment, probably because the binding between the Fabs and their targets is very transient and dynamic
(Hayashi-Takanaka et al. 2011; Sato et al. 2013; Stasevich et al. 2014; Kimura et al. 2015).

\section{Imaging, data quantification, and interpretation}

Embryos were imaged on a spinning-disk confocal microscope as previously described (Farrell et al. 2012), and the images were analyzed using Volocity 6 (Perkin Elmer). When an individual repetitive sequence was imaged, the $Z$-scale was selected based on the TALE-light signals (usually 3-4 $\mu \mathrm{m}$ ), and the step size in the $Z$-axis was $0.5 \mu \mathrm{m}$ for fixed samples and $1 \mu \mathrm{m}$ for live embryos. All of the images in one experiment were acquired and processed using identical microscopic settings. In the TALE-light staining experiments, a single optic section across the indicated repetitive loci was shown.

For the quantification of HP1a recruitment, optic sections containing the specific genomic loci were projected and corrected for photobleaching in Volocity. In each experiment, three to seven embryos and five to seven nuclei in each embryo were selected for quantification. In each nucleus, the mean intensity of GFPHP1a at the TALE-light-positive loci was divided by that at a control locus in that same nucleus to calculate the fold enrichment (Supplemental Fig. S3C). The mean and SD of the calculated fold enrichment in nuclei from different embryos were plotted against time, and then nonlinear regression analysis was performed using Prism (GraphPad).

The results shown in this study were obtained with injected recombinant GFP-HP1a protein. We also performed the experiments with GFP-HP1a expressed from a transgene, and the results were identical. HP1a functions as a dimer, and thus a fraction of the injected GFP-HP1a mutant proteins could form heterodimers with the endogenous wild-type HP1a. The possible existence of the heterodimer pool complicated the experimental scenario but did not influence the interpretation of the contributions of different domains to the initial recruitment of HP1a.

\section{Acknowledgments}

We thank the members of the O'Farrell laboratory, especially Tony Shermoen and Hansong Ma, for continuous support and useful suggestions. We thank Angela Andersen, William Sullivan, Geeta Narlikar, Blake Riggs, Barbara Panning, and Hiten Madhani for their comments on the manuscript; the University of California at San Francisco ES Cell Targeting Core for constructing the TALE-lights; Zeid Rusan for analyzing the small RNA data; and Daniel Barbash, Hiroshi Kimura, Stavros Lomvardas, Barbara Panning, Patrick Ferree, Katalin Toth, Sarah Elgin, Haruhiko Siomi, William Theurkauf, and William Sullivan for sharing reagents. This research was supported by the University of California at San Francisco Program for Breakthrough Biomedical Research (to K.Y.), the University of California at San Francisco Clinical and Translational Science Institute-Strategic Opportunities Support Program (to K.Y.), and National Institutes of Health grant GM037193 (to P.H.O).

\section{References}

Anton T, Bultmann S, Leonhardt H, Markaki Y. 2014. Visualization of specific DNA sequences in living mouse embryonic stem cells with a programmable fluorescent CRISPR/Cas system. Nucleus 5: 163-172.

Beisel C, Paro R. 2011. Silencing chromatin: comparing modes and mechanisms. Nat Rev Genet 12: 123-135. 
Blythe SA, Wieschaus EF. 2015. Zygotic genome activation triggers the DNA replication checkpoint at the midblastula transition. Cell 160: 1169-1181.

Brasher SV, Smith BO, Fogh RH, Nietlispach D, Thiru A, Nielsen PR, Broadhurst RW, Ball LJ, Murzina NV, Laue ED. 2000. The structure of mouse HP1 suggests a unique mode of single peptide recognition by the shadow chromo domain dimer. EMBO J 19: 1587-1597.

Canzio D, Larson A, Narlikar GJ. 2014. Mechanisms of functional promiscuity by HP1 proteins. Trends Cell Biol 24: 377-386.

Chen B, Gilbert LA, Cimini BA, Schnitzbauer J, Zhang W, Li GW, Park J, Blackburn EH, Weissman JS, Qi LS, et al. 2013. Dynamic imaging of genomic loci in living human cells by an optimized CRISPR/Cas system. Cell 155: 1479-1491.

Chiolo I, Minoda A, Colmenares SU, Polyzos A, Costes SV, Karpen GH. 2011. Double-strand breaks in heterochromatin move outside of a dynamic HPla domain to complete recombinational repair. Cell 144: 732-744.

Deng W, Shi X, Tjian R, Lionnet T, Singer RH. 2015. CASFISH: CRISPR/Cas9-mediated in situ labeling of genomic loci in fixed cells. Proc Natl Acad Sci 112: 11870-11875.

Duxin JP, Dewar JM, Yardimci H, Walter JC. 2014. Repair of a DNA-protein crosslink by replication-coupled proteolysis. Cell 159: 346-357.

Edgar BA, O'Farrell PH. 1990. The three postblastoderm cell cycles of Drosophila embryogenesis are regulated in G2 by string. Cell 62: 469-480.

Elgin SC, Reuter G. 2013. Position-effect variegation, heterochromatin formation, and gene silencing in Drosophila. Cold Spring Harb Perspect Biol 5: a017780.

Fadloun A, Eid A, Torres-Padilla ME. 2013. Mechanisms and dynamics of heterochromatin formation during mammalian development: closed paths and open questions. Curr Topics Dev Biol 104: 1-45.

Farrell JA, O'Farrell PH. 2014. From egg to gastrula: how the cell cycle is remodeled during the Drosophila mid-blastula transition. Annu Rev Genet 48: 269-294.

Farrell JA, Shermoen AW, Yuan K, O'Farrell PH. 2012. Embryonic onset of late replication requires $\mathrm{Cdc} 25$ down-regulation. Genes Dev 26: 714-725.

Ferree PM, Barbash DA. 2009. Species-specific heterochromatin prevents mitotic chromosome segregation to cause hybrid lethality in Drosophila. PLoS Biol 7: e1000234.

Gu T, Elgin SC. 2013. Maternal depletion of Piwi, a component of the RNAi system, impacts heterochromatin formation in Drosophila. PLoS Genet 9: e1003780.

Guzzardo PM, Muerdter F, Hannon GJ. 2013. The piRNA pathway in flies: highlights and future directions. Curr Opin Genet Dev 23: 44-52.

Hathaway NA, Bell O, Hodges C, Miller EL, Neel DS, Crabtree GR. 2012. Dynamics and memory of heterochromatin in living cells. Cell 149: 1447-1460.

Hayashi-Takanaka Y, Yamagata K, Wakayama T, Stasevich TJ, Kainuma T, Tsurimoto T, Tachibana M, Shinkai Y, Kurumizaka H, Nozaki N, et al. 2011. Tracking epigenetic histone modifications in single cells using Fab-based live endogenous modification labeling. Nucleic Acids Res 39: 6475-6488.

Holt LJ, Krutchinsky AN, Morgan DO. 2008. Positive feedback sharpens the anaphase switch. Nature 454: 353-357.

Jachowicz JW, Santenard A, Bender A, Muller J, Torres-Padilla ME. 2013. Heterochromatin establishment at pericentromeres depends on nuclear position. Genes Dev 27: 2427-2432.
Kimura H, Hayashi-Takanaka Y, Stasevich TJ, Sato Y. 2015. Visualizing posttranslational and epigenetic modifications of endogenous proteins in vivo. Histochem Cell Biol 144: 101-109.

Krassovsky K, Henikoff S. 2014. Distinct chromatin features characterize different classes of repeat sequences in Drosophila melanogaster. BMC Genomics 15: 105.

Lee MT, Bonneau AR, Giraldez AJ. 2014. Zygotic genome activation during the maternal-to-zygotic transition. Annu Rev Cell Dev Biol 30: 581-613.

Levsky JM, Singer RH. 2003. Fluorescence in situ hybridization: past, present and future. J Cell Sci 116: 2833-2838.

Li XY, Harrison MM, Villalta JE, Kaplan T, Eisen MB. 2014. Establishment of regions of genomic activity during the Drosophila maternal to zygotic transition. Elife 3: e03737.

Lindhout BI, Fransz P, Tessadori F, Meckel T, Hooykaas PJ, van der Zaal BJ. 2007. Live cell imaging of repetitive DNA sequences via GFP-tagged polydactyl zinc finger proteins. Nucleic Acids Res 35: e107.

Lu BY, Ma J, Eissenberg JC. 1998. Developmental regulation of heterochromatin-mediated gene silencing in Drosophila. Development 125: 2223-2234.

Ma H, Reyes-Gutierrez P, Pederson T. 2013. Visualization of repetitive DNA sequences in human chromosomes with transcription activator-like effectors. Proc Natl Acad Sci 110: 21048-21053.

Mendez DL, Kim D, Chruszcz M, Stephens GE, Minor W, Khorasanizadeh S, Elgin SC. 2011. The HP1a disordered C terminus and chromo shadow domain cooperate to select target peptide partners. Chembiochem 12: 1084-1096.

Mendez DL, Mandt RE, Elgin SC. 2013. Heterochromatin protein 1a (HP1a) partner specificity is determined by critical amino acids in the chromo shadow domain and C-terminal extension. J Biol Chem 288: 22315-22323.

Miyanari Y, Ziegler-Birling C, Torres-Padilla ME. 2013. Live visualization of chromatin dynamics with fluorescent TALEs. Nat Struct Mol Biol 20: 1321-1324.

Padeken J, Heun P. 2014. Nucleolus and nuclear periphery: velcro for heterochromatin. Curr Opin Cell Biol 28C: 54-60.

Robinett CC, Straight A, Li G, Willhelm C, Sudlow G, Murray A, Belmont AS. 1996. In vivo localization of DNA sequences and visualization of large-scale chromatin organization using lac operator/repressor recognition. J Cell Biol 135: 1685-1700.

Rudolph T, Yonezawa M, Lein S, Heidrich K, Kubicek S, Schafer C, Phalke S, Walther M, Schmidt A, Jenuwein T, et al. 2007. Heterochromatin formation in Drosophila is initiated through active removal of H3K4 methylation by the LSD1 homolog SU (VAR)3-3. Mol Cell 26: 103-115.

Saito K, Nishida KM, Mori T, Kawamura Y, Miyoshi K, Nagami T, Siomi H, Siomi MC. 2006. Specific association of Piwi with rasiRNAs derived from retrotransposon and heterochromatic regions in the Drosophila genome. Genes Dev 20: 2214-2222.

Sato Y, Mukai M, Ueda J, Muraki M, Stasevich TJ, Horikoshi N, Kujirai T, Kita H, Kimura T, Hira S, et al. 2013. Genetically encoded system to track histone modification in vivo. Sci Rep 3: 2436.

Schwaiger M, Kohler H, Oakeley EJ, Stadler MB, Schubeler D. 2010. Heterochromatin protein 1 (HP1) modulates replication timing of the Drosophila genome. Genome Res 20: 771-780.

Segal DJ, Dreier B, Beerli RR, Barbas CF III. 1999. Toward controlling gene expression at will: selection and design of zinc finger domains recognizing each of the $5^{\prime}$-GNN-3' DNA target sequences. Proc Natl Acad Sci 96: 2758-2763.

Sexton T, Yaffe E. 2015. Chromosome folding: driver or passenger of epigenetic state? Cold Spring Harb Perspect Biol 7: a018721. 
TALE-light illuminates heterochromatin formation

Shermoen AW, McCleland ML, O'Farrell PH. 2010. Developmental control of late replication and S phase length. Curr Biol 20: 2067-2077.

Stasevich TJ, Hayashi-Takanaka Y, Sato Y, Maehara K, Ohkawa Y, Sakata-Sogawa K, Tokunaga M, Nagase T, Nozaki N, McNally JG, et al. 2014. Regulation of RNA polymerase II activation by histone acetylation in single living cells. Nature 516: 272-275.

Thanisch K, Schneider K, Morbitzer R, Solovei I, Lahaye T, Bultmann S, Leonhardt H. 2014. Targeting and tracing of specific DNA sequences with dTALEs in living cells. Nucleic Acids Res 42: e38.

Usakin L, Abad J, Vagin VV, de Pablos B, Villasante A, Gvozdev VA. 2007. Transcription of the 1.688 satellite DNA family is under the control of RNA interference machinery in Drosophila melanogaster ovaries. Genetics 176: 1343-1349.

Vlassova IE, Graphodatsky AS, Belyaeva ES, Zhimulev IF. 1991. Constitutive heterochromatin in early embryogenesis of Drosophila melanogaster. Mol Gen Genet 229: 316-318.
Waters JC. 2009. Accuracy and precision in quantitative fluorescence microscopy. J Cell Biol 185: 1135-1148.

Wei KH, Grenier JK, Barbash DA, Clark AG. 2014. Correlated variation and population differentiation in satellite DNA abundance among lines of Drosophila melanogaster. Proc Natl Acad Sci 111: 18793-18798.

Yuan K, Shermoen AW, O'Farrell PH. 2014. Illuminating DNA replication during Drosophila development using TALElights. Curr Biol 24: R144-R145.

Zemach A, McDaniel IE, Silva P, Zilberman D. 2010. Genomewide evolutionary analysis of eukaryotic DNA methylation. Science 328: 916-919.

Zhao T, Eissenberg JC. 1999. Phosphorylation of heterochromatin protein 1 by casein kinase II is required for efficient heterochromatin binding in Drosophila. J Biol Chem 274: 15095-15100.

Zhao T, Heyduk T, Eissenberg JC. 2001. Phosphorylation site mutations in heterochromatin protein 1 (HP1) reduce or eliminate silencing activity. J Biol Chem 276: 9512-9518. 


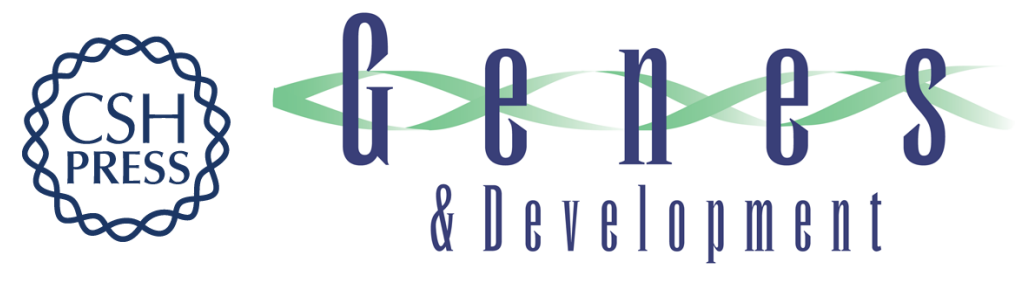

\section{TALE-light imaging reveals maternally guided, H3K9me2/3-independent emergence of functional heterochromatin in Drosophila embryos}

Kai Yuan and Patrick H. O'Farrell

Genes Dev. 2016, 30: originally published online February 25, 2016

Access the most recent version at doi:10.1101/gad.272237.115

Supplemental Material

References

Creative

Commons

License

Email Alerting

Service
http://genesdev.cshlp.org/content/suppl/2016/02/23/gad.272237.115.DC1

This article cites 51 articles, 20 of which can be accessed free at: http://genesdev.cshlp.org/content/30/5/579.full.html\#ref-list-1

This article is distributed exclusively by Cold Spring Harbor Laboratory Press for the first six months after the full-issue publication date (see http://genesdev.cshlp.org/site/misc/terms.xhtml). After six months, it is available under a Creative Commons License (Attribution-NonCommercial 4.0 International), as described at http://creativecommons.org/licenses/by-nc/4.0/.

Receive free email alerts when new articles cite this article - sign up in the box at the top right corner of the article or click here.

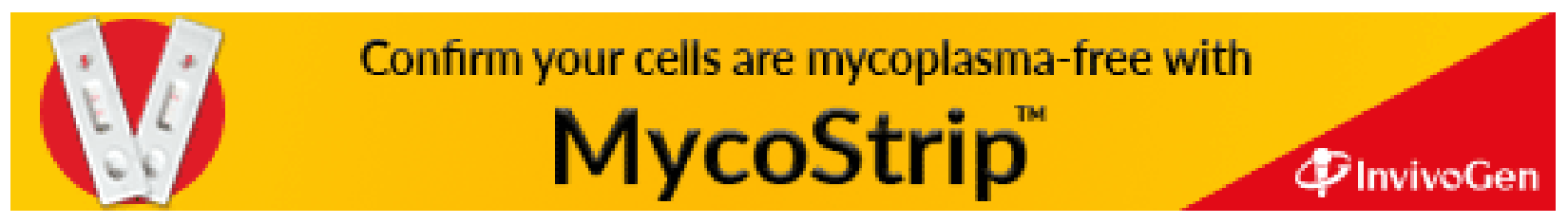

